\title{
Papel de los establecimientos de enseñanza en el perfeccionamiento de los funcionarios europeos $\left(^{*}\right)$
}

\section{LIMITES DE ESTA PONENCIA}

La perfecta sistemática de los temas incluídos en el programa de este Coloquio Internacional sobre la formación y perfeccionamiento de los funcionarios europeos, delimita con gran precisión el tema de esta ponencia. Queda fuera de nuestro estudio, por ser objeto de otras ponencias, el concepto de funcionario europeo y todo lo relativo a la formación previa a su nombramiento, así como también las tareas que en cuanto al perfeccionamiento de los funcionarios europeos incumben a las administraciones europeas y na-. cionales.

Al hablar del perfeccionamiento de los funcionarios nos referimos, por tanto, a la formación posterior al acto del nombramiento. La formación del funcionario no termina naturalmente en el momento en que se nombra. La formación es la labor de todá su vida, si no quiere perder gradualmente eficacia y ver atrofiados sus conocimientos y facultades profesionales. Un funcionario que abandonara sus estudios profesionales, que d́ejara de mantener al dia sus conocimientos técnicos, que se desentendiera de la obligación de conocer los nuevos métodos de trabajo y los últimos avances de

(*) Ponencia presentada por el autor al Coloquio Internacional organizado por la Universidad del Sarre. 
la ciencia administrativa, caería fácilmente en la rutina y entorpecería la marcha del servicio que tenga encomendado.

\section{Servicio aCtivo y CURSOS DE PERfECCIONAMIENTo}

Si la formación es tarea de toda la vida profesional del funcionario y si, a la vez, el funcionario en activo tiene su tiempo consagrado al ejercicio de su función, salta a la vista la necesidad de coordinar estas dos exigencias; de forma que ni se abandone la idea del perfeccionamiento de los funcionarios ni trate de lograrse en forma que haga padecer gravemente la atención y asiduidad que han de poner en el ejercicio de sus cargos.

De aquí que prima facie se nos ofrezcan dos modos de organización de los cursos de perfeccionamiento: o como cursos nocturnos, después de las horas de oficina y compatibles, por tanto, con el desempeño de los cargos de los funcionarios, o bien como cursos de vacaciones para aprovechar este periodo con tareas que por ser distintas de las que habitualmente realiza el funcionario le suponen un descanso y a la vez le brindan ocasión de mejorar su for- · mación profesional. Si se quiere respetar la absoluta intangibilidad de los períodos de vacaciones, pueden también organizarse los cursos de perfeccionamiento en forma intensiva y a base de la concesión de licencias o permisos a los funcionarios durante el tiempo en que dichos cursos se celebren.

En todo caso, el modo más eficaz para el perfeccionamiento de los funcionarios consiste en la realización de cursos de ampliación de estudios en los que se combinen las materias teóricas y prácticas con arreglo a un programa cientificamente elaborado.

LOS ESTABLECIMIENTOS DOCENTES Y LOS CURSOS DE PERFECCIONAMIENTO

Si de cursos de estudios se trata, es lógico que el papel que incumbe a los establecimientos de enseñanza sea primordial. Aparte de la labor de perfeccionamiento de los funcionarios europeos que 
pueden correr a cargo de las administraciones europeas y nacionales (analizadas en otra ponencia), las Universidades, las Escuelas de Administración, otros centros docentes especializados y los Institutos de Estudios europeos; están llamados a jugar un importante papel en el perfeccionamiento de los funcionarios europeos.

Será oportuno analizar por separado las actuales realizaciones que ofrece cada tipo de establecimientos de enseñanza en cuanto a cursos de perfeccionamiento de funcionarios se refiere, y las posibilidades que se advierten cara al futuro para ampliar su labor formativa del funcionario europeo.

\section{A) Universidades.}

La Universidad, alma mater de la cultura europea e institución en la que culminan los saberes humanos, no puede estar ausente en la tarea de formación de los funcionarios europeos. Ahora bien, su misión en cuanto a dicha formación se refiere, ha de ser precisada con algún detenimiento.

\section{MOdOS DE LA ACCIÓN FORMATIVA DE LOS FUNCIONARIOS}

La formación del funcionario puede realizarla la Universidad de un modo directo y deliberado o de un modo indirecto, es decir, estableciendo cursos específicos destinados a la formación de funcionarios o simplemente contando entre sus alumnos a funcionarios públicos en los cursos ordinarios de cualquier Facultad universitaria, y especialmente en aquellas que, como las de Derecho, Ciencias Políticas o Economía, guardan mayor relación con las profesiones burocráticas. Teniendo en cuenta que gran parte de los funcionarios públicos proceden de las aulas universitarias, es evidente la influencia formativa que la Universidad tiene sobre los funcionarios. Pero esta simple influencia indirecta no es bastante, y muchos proponen que la Universidad se ocupe directamente dế perfeccionamiento de los funcionarios, bien creando Facultades o Escuelas consagradas específicamente a dichas tareas-como son 
las Facultades de Administración que existen en muchas Universidades norteamericanas -0 bien organizando cursos nocturnos o cursos especiales abreviados para el perfeccionamiento de los funcionarios.

Al presentarse la posibilidad de una intervención universitaria directa en el perfeccionamiento de los funcionarios, se suscita el tema muy debatido de cuál sea la verdadera misión de la Universidad, y a la vez se presenta también el problema aún no resuelto del predominio de la cultura general o de la especialización en la formacion de los funcionarios.

\section{MISIÓN DE LA UNIVERSIDAD}

No vamos a extendernos aquí en consideraciones sobre la misión de la Universidad. Nos podemos remitir a los luminosos escritos del Cardenal Newman, Scheler, Jaspers, Azevedo, Barquer, Basadre, Belenhradek, Lambert, Wenzl, Moberly, Ladeuze y entre los españoles, Ibáñez Martín, Rodríguez Fornos, Ortega, Albareda, Lain y Alvaro d'Ors. En resumen, se asigna ordinariamente a la Universidad este triple cometido formativo: formación cultural, formación moral y formación profesional. Las tres direcciones de la labor formativa universitaria son necesarias, hasta el punto de que el olvido de cualquiera de ellas vendría a mutilar la misión de la Universidad. La Universidad no puede contentarse con ser una oficina expendedora de títulos profesionales; ha de ser también un centro difusor de la ciencia y la cultura que transmita : sus alumnos los conocimientos y valores fundamentales de la cultura occidental. Pero tampoco puede contentarse con formar hombres cultos; ha de aspirar además a formar hombres rectos, adornados de virtudes morales. Un profesional culto sin virtudes morales no será nunca un buen funcionario. Un funcionario ha de vivir la lealtad, el espiritu de servicio, la probidad, una serie, en fin, de virtudes morales sin ias que la función púbiica no estaría debidamente atendida. 


\section{Cultura general Y especialización}

Sin embargo, no siempre se han mantenido con la debida ponderăción estas tres direcciones de la formación universitaria. Dos de ellas se hallan frecuentemente en pugna, a saber, la formación profesional y la formación cultural. Algunos han pretendido que la Universidad no debe formar profesionales, sino simplemente hombres cultos en las distintas ramas del saber, y que la profesión es algo que debe aprenderse después en Escuelas especiales. Por el contrario, según otras, la función primaria de la Universidad consiste en la formación profesional $y$ ha de organizar cursos tan especializados como la complejidad de las profesiones modernas exija, para que de sus aulas se pueda salir profesionalmente formados, aunque esta especialización obligue a apartarse del tronco común de la cultura general.

Entiendo que tan errónea es una postura como la otra y que la Universidad debe a la vez mantener en los programas de sus cursos un mínimo de materias de cultura general y debe también llegar a la especialización. Por ello, no creo que sea ajeno a la Universidad el organizar cursos especiales de perfeccionamiento de funcionarios, si bien estos cursos, para mantener una altura universitaria, no deberán ceñirse a lo estrictamente profesional, sino que habrán de ser ocasión para que los funcionarios eleven también su nivel cultural. No pueden darse en las aulas universitarias cursos para el mero adiestramiento profesional, sino que, junto a esa tarea, hay que abrir el espíritu de los funcionarios a los grandes temas culturales del momento presente.

\section{TEORfA PRACTICA}

También se tha presentado la escisión entre la teoría y la práctica, olvidando que la theoría, como ya enseñó Aristóteles, es la forma suprema de la praxis. Tan verdadera es la sentencia aristotélica, que siempre el mejor instrumento para una buena práctica 
es la posesión intelectual de una buena teoría. Toda la Mecánica práctica posterior al siglo xvIr no hubiera sido posible sin la obra teorética de Galileo; y el ingeniero que maneja hoy con fines industriales la energía atómica tiene su razón de ser en la labor puramente intelectual a que Alberto Einstein se entrego, sin pensar en posibles aplicaciones ingenieriles, durante los dos prịmeros decenios de nuestro siglo.

Por ello los cursos universitarios de perfeccionamiento están plenamente justificados. La Universidad, sede de enseñañzas fundamentalmente teóricas, tiene mucho que decir a los funcionarios que no pueden despreciar la teorla sin riesgo de perder su rango social y profesional reduciéndose a la triste condición de practicones. Unos conocimientos teóricos de Derecho administrativo, Derecho internacional y Derecho comparado, por ejemplo, son ingredientes imprescindibles de todo programa de ampliación de estudios de los funcionarios europeos.

Punto de vista de los Congresos de Ciencias Administrathinas

En este sentido se expresó el Instituto Internacional de Ciencias Administrativas en su última "round-table" celebrada en Oxford en el mes de julio, al llegar a la conclusión de que debe ponerse de relieve "la necesidad tanto de la teoría como de la práctican. Y que cabe compaginar las dos formaciones, la cultura general y la especialización, "asignando tanto tiempo a la una como a la otra, y precediendo siempre la formación general a la especialización. En suma, nada de exclusivismo. En cuanto al carácter general de la enseñanza, la gran mayoría de los delegados estima y comenta con convicción que la primacía debe recaer sobre la formación general y la amplitud de espíritu que la misma fomenta. No se trata, bien entendido, de rechazar la especialización, sino de recurrir a ésta lo más tarde posiblen.

En este orden de ideas se expresó también el profesor Seeldráyers en su "rapport" presentado al Congreso Internacional de Cier:ias Administrativas de Estambul en 1953, señalando que el per- 
feccionamiento de los funcionarios debe afectar a la vez a la cultura general y a los conocimientos técnicos y profesionales. Acerca de uno y otro aspecto se formulaba el citado profesor una serie de cuestiones que estimo interesante reproducir aquí :

\section{Puntos a dilucidar}

\section{Perfeccionamiento de la cultura general}

Es extremadamente importante definir el grado y naturaleza de la cultura general que pueda ser exigida a los funcionarios superiores. ¿Trátase de conocimientos literarios y artísticos? Por el contrario, ¿trátase exclusivamente de una cultura general de la especialidad $? \dot{i}$ Conviene, llegado el caso, exigir de los funcionarios una formación a la vez literaria y especializada en el dominio de la técnica que serán llamados a aplicar?

Perfeccionamiento de los conocimientos técnicos-y profesionales

¿Cuáles son estos conocimientos?

En lo que concierne a los funcionarios superiores, sería útil examinar si estos conocimientos deben comportar, a más de los de su especialización, un bagaje serio en materia de Derecho público y administrativo, en materia de economía política, en materia financiera, y en materia de organización administrativa. ¿Se puede admitir, por ejemplo, que un médico llegue a las funciones administrativas superiores en un Ministerio de Salud Pública si, fuera de sus conocimientos relativos al arte de curar, no posee una formación general especializada de administrador? En una hipótesis semejante, ¿cuál es el mínimo de conocimientos que comporta el ejercicio de la función de administrador?

\section{REALizaciones aCtuales}

Las realizaciones que tenemos a la vista en orden a la labor de las Universidades para el perfeccionamiento de los funcionarios son fundamentalmente las siguientes : 


\section{A) Universidades americanas.}

De una parte, la creación de Facultades o Escuelas universitarias destinadas especificamente a la enseñanza de las Ciencias administrativas o Facultades llamadas de Administración Pública. Así han hecho varias Universidades norteamericanas ; por ejemplo, la University of Southern California, que creó en 1928 una "School of Public Administration". También la Universidad de New York ha creado una Facultad de Administración Pública; el 70 por 100 de cuyos alumnos ingresan al servicio de la Administración. En €sta última Facultad se dan cursos de Administración internacional, a los que asisten gran número de funcionarios de las Naciones Unidas.

Estos cursos ofrecen especial interés en relación con la materia que nos ocupa del perfeccionamiento de las funcionarios europeos, dado su carácter de funcionarios internacionales.

En otros casos esas Facultades o Escuelas no sólo preparan para el desempeño de funciones públicas, sino también para el ejercicio de cargos administrativos en empresas mercantiles e industriales. Tal es el caso del "College of Business and Public Administration" de la Universidad de Maryland.

En uno y otro caso la formación profesional universitaria va encaminada a la capacitación para el ejercicio de funciones administrativas. Se trata, pues, de la enseñanza de la Administración pública o privada como carrera universitaria. Este fenómeno, perfectamente concorde con las características de las Universidades norteamericanas, muy diferentes de la Universidad tradicional europeă, ejerce ya gran influencia en América del Sur. Concretamente en Colombia acaba de crearse una nueva Universidad en Pamplona, una de cuyas Facultades será de Administración fública y privada.

Ello nos pone de relieve que la Universidad no tiene el mismo contenido en Europa que en América. Incluso !a Universidac inglesa, tan distinła de la nazoleónica, y que sirvín de pấón as las Universidades alemanas o francesas, are a la de Yale o de 
Harvard, en donde existen disciplinas orientadas directamente hacia la Técnica como tal.

\section{B) Universidad Continental.}

En Francia, en cambio, como consigna M. Guy Debeyre, Decano de la Facultad de Derecho de Lille, la Universidad "no se ha interesado por una preparación directa y concreta para las funcio. nes administrativas, ya se tratase del sector privado o del sector público. Las diversas Facultades que componen una Universidad no son Escuelas profesionalesı.

Esta carencia ha tenido como efecto la creación de organismos extrauniversitarios, tales como la Escuela de Administración de Negocios por la Industria del Norte de Francia, y la Escuela Nacional de Administración por la Presidencia del Consejo, fuera de la Universidad.

\section{CARÁCTER DE LOS ESTUdIOS ADMINISTRATIVOS}

Ante este contraste entre la Universidad profesional y técnica americana y la Universidad tradicional europea, cabe preguntarse si realmente la ciencia administrativa es una disciplina de carácter académico. Se ha dicho que la Administración no puede constituir disciplina universitaria, puesto que no basta para formar un hombre. Constituye más bien un arte o incluso una ciencia instrumental propia de una enseñanza especializada extrauniversitaria. Otra objeción al carácter universitario de los estudios administrativos és que la teoría de la Administración es a la vez breve y fácil, mientras que la práctica es cuestión de toda la vida y la Universidad no puede comunicar este conocimiento pragmático.

Frente a las anteriores razones está una consideración realista derivada de las necesidades económicas y de otras circunstancias concretas. Se admite, sin embargo, que las circunstancias-exigüidad de medios financieros, número restringido de alumnos, variedad de casos-puedan llevar, en ciertos países, a recurrir a una en- 
señanza; general o especializada, según los casos, en el seno de las Universidades existentes.

\section{CURSOS UNIVERSITARIOS DE PERTECCIONAMIENTO}

Así ha ocurrido en España, donde se han organizado cursos de perfeccionamiento de los funcionarios superiores municipales en distintas Universidades en conexión con el Instituto de Estudios de Administración Local. Gracias a estos cursos en las Universidades provinciales se ha evitado los gastos de deplazamiento de los funcionarios a la capital para seguir los cursos de perfeccionamiento urganizados en la sede del Instituto de Estudios de Administración Local de Madrid. Los cursos han tenido lugar en las aulas universitarias y han corrido a cargo de profesores de la Facultad de Derecho $y$ de altos funcionarios municipales. Esta experiencia of rece indudable interés, especialmente desde el punto de vista económico, por suponer una indudable reducción de gastos. Quizá pueda servir de pauta para la organización por el Consejo de Europa u otras Administraciones europeas de cursos semejantes en distintas Universidades continentales, especialmente de las ciudades donde radique el mayor número de funcionarios europeos.

\section{B) Escuelas de Adminisíración.}

En el orden de las realizaciones actuales hemos de ocuparnos especialmente de "l'Ecole National d'Administration" de París y del "Institut Egyptien d'Administration" fundado por las Nacicnes Unidas.

\section{Ecole National d'Administration}

Como es sabido, esta Escuela, creada en octubre de 1945 y dependiente de la Presidencia del Consejo, admite dos clases de alumnos: estudiantes y funcionarios.

Por lo que respecta a los funcionarios, que es lo que interesa a nuestro propósito, se exige que su edad esté comprendida entre 
los veinticuatro y los treinta años, y que cuenten con cuatro años de servicio activo en los cuadros de la Administración pública. Es de advertir que, para ingresar como alumno en la Escuela, se exige el tener nacionalidad francesa, y los extranjeros sólo pueden acudir a la Escuela en calidad de oyentes. Ello implica que, hoy por hoy, solamente podrian ser alumnos de dicha Escuela aquellos funcionarios europeos que posean la nacionalidad francesa.

Los alumnos funcionarios pasan a la situación de "detachemen", y se les compensa la disminución que en su retribución experimentan por pasar a esa situación, sin que dejen de avanzar en sus escalafones.

Con independencia de las retribuciones antes dichas, se paga a los alumnos el viaje hasta París, y a los que verifican las prácticas de primer año se les abona una indemnización de 240.000 francos a los casados y 112.000 a los solteros.

Los alumnos funcionarios que tengan hijos y que no encuentren vivienda en el lugar a donde se trasladan para verificar sus estudios reciben 175 francos diarios.

\section{Nombramientos al salir de la Escuela}

Sobre la base de la precedencia que a cada alumno asignan las calificaciones obtenidas al terminar los estudios, se les destina al Cuerpo por ellos elegido con la categoría de entrada en él. Los que fueran funcionarios antes de su ingreso en la Escuela pierden todo derecho a volver al Cuerpo de origen, pero se les computa el tiempo de servicio en él a efectos pasivos y, en tanto su retribución en el nuevo Cuerpo sea menor que la que tenía en el anterior, se les abona la diferencia entre ambas.

La Escuela consta de cuatro secciones: Administración General, Administración Económica y Financiera, Administraciọn Social y Asuntos Exteriores.

La Escuela funciona en régimen de externado. Las enseñanzas duran tres años:' el primero de prácticas, el segundo de estudios y el tercero mixto. 


\section{"Centre des Hautes Etudes Administratives"}

Junto a la Escuela Nacional de Administración existe el "Centre des Hautes Etudes Administratives" que, desde su origen, se halla vinculado a la Escuela : mismo Director, mismo Consejo de Administración y mismos locales.

Conforme al artículo 2. ${ }^{\circ}$ de un Decreto de 9 de octubre de 1945, "el Centro de Altos Estudios Administrativos tiene por objeto: $1 .^{\circ}$, a título principal, perfeccionar la cultura de funcionarios que tengan ya experiencia de la vida administrativa, tenerles al corriente de las cuestiones actuales y darles ocasión de completar sus conocimientos especiales mediante el estudio de problemas nuevos; 2., a título complementario, dar una formación administrativa a personas que hayan realizado ya sus pruebas en actividades privadas y que deseen consagrarse al servicio del Estado en los empleos que puedan serles abiertos").

Como subraya M. Bourdeau de Fontenay, "el Centro está abierto, por tanto; a personas del sector privado, que pueden participar en sus cursos a título de oyentes libres; aquí debe señalarse una evolución con respecto a lo que parece indicar una interpretación literal del texto: no se trata solamente de formar personas que tengan actividades privadas $y$ deseen consagrarse ai servicio del Estado; se trata también de dar a los cuadros del mundo de los negocios las mismas condiciones que a los cuadros de la función pública. Al acoger un número creciente de oyentes libres, el Centro permite, además, a los funcionarios que siguen sus cursos, entrar en contacto no sólo con otras Administraciones o servicios, sino incluso con futuros dirigentes de empresas públicas o privadas".

Conforme a los textos de 9 de octubre de 1945, "los oyentes que hubieran ejecutado de forma juzgada satisfactoria el conjunto de trabajos previstos" obtendrán un diploma que "podrá ser tenido en cuenta" en los nombramientos para empleos superiores de la Administración.

Este Centro es también de carácter oficial y representa una suerte de Estado Mayor de la Administración francesa. Aspira a formar 
al personal directivo de los cuadros burocráticos. Para la entrada en este Centro se exige a los funcionarios hallarse comprendidos entre los treinta y los cuarenta y cinco años y contar con seis años de servicio activo. Los cursos duran cuatro meses y ocupan dos dias y. medio por semana. El método de trabajo consiste en consagrar cada sección al estudio de una materia determinada, a ser posible temas de actualidad. Los cursos no están dirigidos por un personal docente permanente, sino por uno o varios directores de trabajo. El curso consta de tres periodos : las tres primeras semanas se consagran a la exposición de los temas elegidos; luego, durante cinco - seis semanas, se estudian los distintos aspectos del tema en pequeños grupos de ocho personas, como máximo; por último, se realiza una labor de sintesis con los datos aportados por cada uno de los grupos y se redacta un "rapport" final. Para la recogida de datos durante el segundo período se realizan encuestas, entrevistas, viajes, etc.

\section{Otros establecimientos franceses}

En Francia existen otros Centros directamente relacionados con la formación y perfeccionamiento de los funcionarios, tales como el Instituto de Estudios Políticos, el "Centre de Recherches Administratives", el "Institut Thecnique des Administrations Publiques".

Este último fué creado en 1947 por un grupo de funcionarios y. personalidades. Es, a la vez, un Centro de investigaciones e información y de perfeccionamiento de los funcionarios. Organiza grupos de estudios, cursos de conferencias y "stages de formation» de tres semanas de duración.

\section{"Administrative Staff College"}

Es un Centro de carácter privado creado en Inglaterra en julio de 1945 con el fin de perfeccionar la formación de funcionarios públicos y altos empleados de empresas privadas que cuenten con una larga experiencia en el ejercicio de sus profesiones. 
Tiene su sede en Henley-on-Thames y no está vinculado a ninguna Universidad ni centro oficial. Se ha procurado emplazarlo en un lugar igualmente accesible a los núcleos industriales, mercantiles y administrativos del país.

La duración del curso es de tres meses y absorbe enteramente la jornada de los participantes.

El College no se propone exponer una dirección administrativa específica ni demiostrar un conjunto de "pratiques approuvées", sino dar ocasión a funcionarios y hombres de empresa experimentados para que cambien impresiones sobre los resultados de sus experiencias, y realizar un examen crítico de sus organizaciones de trabajo y de sus prácticas. Como escribió Sir Hetherington, "en agosto de 1955 serán ya casi 1.300 personas, hombres y mujeres, las que habrán seguido los cursos. Alrededor de un 10 por 100 de los estudiantes son originarios de países de ultramar, y los demás vienen del Reino Unidon..

El método de trabajo consiste en dividir los aluminos en pequeños grupos llamados "syndicates", con un máximo de nueve o diez participantes, convenientemente dosificados, de forma que en un mismo grupo asistan funcionarios de la Administración central y local, de la Banca y de las principales ramas de la industria y el comercio. La labor de estos grupos pasa por distintas fases : una primera, de cambio de impresiones para precisar conceptos y aclarar ideas ; una segunda, en la: que se realizan encuestas, entrevistas a expertos ajenos al College, etc., y una tercera fase, en la que se elabora el "rapport» final. El tiempo consagrado a cada tema está estrictamente limitado; diariamente cada grupo ha de resumir por escrito el resultado de sus deliberaciones para que pueda ser examinado por otros grupos. De aquí que se haya fijado como unidad de trabajo una hora y media. La jornada comprende tres de dichos períodos, dos por la mañana y uno por la tarde. Además de estos tres periodos, hay una sección conjunta para el examen de los urapports" de los distintos equipos. La sesión de presentación de "rapports" suele durar unas tres horas.

Como indica Mr. Noel F. Hall, Principal del "Administrative Staff College», el método de trabajo de Henley "no está llamado a 
sustituir a la enseñanza sistemática, allí donde ésta sea deseable y haya un número suficiente de profesores competentes disponibles. En este caso, probablemente podrían ser formados grupos más numerosos y ser muy diferentes los métodos, aproximándose a los que son puestos en práctica para la formación de hombres y mujeres más jóvenes y que tengan menos experiencia).

Como se ve, los métodos de trabajo del "Centre des Hautes Etudes Administratives" y del "Administrative Staff College" son muy, parecidos. Sin embargo, es preciso subrayar una diferencia importante: que mientras en el College se da un ciclo completo de cursos, según un programa general, idéntico, salvo algunos detalles para todos los grupos, en el "Centre des Hautes Etudes Administratives") se ha adoptado el sistema de señalar una materia concreta para cada grupo.

\section{Otros Centros similares}

En Holanda existe también un Centro semejante al "Administrative Staff College», donde se cursan enseñanzas superiores tanto en Administración pública como en Administración privada. Los cursos se realizan durante cuatro o cinco meses; a razón de dos o tres horas al día. Existen, además, cursos de dos meses para dirigentes de segunda categoría de Administración pública y privada. La formación se basa también en el método de discusiones.

El Instituto Egipcio de Administración Pública, fundado por iniciativa de las Naciones Unidas, exige previamente a sus alumnos una amplia formación de cultura general. En dicho Instituto se cursan estudios de Administración pública, no de Administración privada.

\section{ESTABLECIMIENTOS ESPAÑOLES}

En España no tenemos ningún Centro dedicado exclusivamente al perfeccionamiento de los funcionarios públicos con carácter general. Sin embargo, existen algunos establecimientos que of recen cierto interés. 
a) Escuelas sociales.

En primer lugar, las llamadas Escuelas sociales, dependientes del Ministerio de Trabajo, que tienen por objeto la formación administrativa, juridica y social del personal inscrito en organismos que dependan del Ministerio de Trabajo, de la Delegación Nacional de Sindicatos y de otros organismos sociales. Existe una Escuela Central en Madrid y diversas Escuelas en provincias, atendidas principalmente por profesorado universitario y que utilizan muchas veces para sus clases las aulas de la Facultad de Derecho. Las tareas docentes de la Escuela consisten en cursos regulados y sistẹmáticos articulados en cuatro grupos de enseñanza : de tipo social, de tipo económico, de tipo jurídico y de tipo histórico-cultural. Las enseñanzas de la Escuela constan de tres cursos completos de duración análoga a los cursos académicos, al final de los cuales se entrega el diploma de "Graduado Social".

b) Escuela Nacional de Administración y Estudios Urbanos.

Para los funcionarios locales existe en el seno del Instituto de Estudios de Administración Local una Escuela Nacional de Administración y Estudios Urbanos, creada por Ley de 6 de septiembre de 1940.

Junto a los cursos de formación que dan acceso a los Cuerpos nacionales de Secretarios, Interventores y Depositarios, la Escuela organiza también cursos de perfeccionamiento para los que ya son funcionarios, cuya aprobación constituye un mérito especial para los concursos de ascenso. Además, la Escuela concede diplomas a quienes siguen los cursos de las diferentes especialidades técnicas de la Administración local o de Urbanismo que se enseña en la Escuela.

Como disciplinas fundamentales de la Sección de Administración local de la Escuela deben citarse : el Derecho de las Entidades locales (que comprende el Derecho municipal y el Derecho provincial) ; la técnica de los Presupuestos y de la Contabilidad administrativa; Documentación y Organización de oficinas, y Haciendas 
locales. En la Sección de Estudios urbanos se enseñan : Generalidades de Urbanismo ; Servicios urbanos ; Legislación y Administración urbanísticas, y. Planes urbanos.

Los cursos de perfeccionamiento han sido seguidos por 204 secretarios, 90 interventores, 20 depositarios y 58 funcionarios administrativos de las Diputaciones y Ayuntamientos.

En algunos de estos cursos, la numerosa asistencia ha hecho necesaria la apertura de Secciones regionales de Estudios en algunas ciudades españolas, que han alcanzado, en algunos momentos, el número de veintidós. Las ciudades que poseen Universidad han sido preferidas para ello. Los cursos se han dado en colaboración con las Facultades de Derecho y los Colegios oficiales de Secretarios, Interventores y. Depositarios de Administración local.

Además, para hacer fácil la asistencia de los funcionarios a los cursos de perfeccionamiento, se ha previsto la concesión por las Corporaciones de permisos con sueldo entero, aparte de las bolsas de estudio que deben conreder reglamentariamente y otras de carácter voluntario, concedidas a veces a sus servidores.

\section{c) Escuela Diplomática.}

Por último, existe en España una Escuela Diplomática creada por un Decreto de 1942.

E1 ingreso se hace por oposición, y una vez en la Escuela, los alumnos siguen un curso de formación y de perfeccionamiento, que comprende : $a$ ), ampliación del estudio de las lenguas; $b$ ), Derecho diplomático y prácticas de Cancillería ; c), Derecho consular y redacción de documentos pủblicos; $d$ ), teoría y práctica de la protección diplomática de los nacionales en el extranjero; $e$ ), Política económica, comercial y técnica de los tratados comerciales ; f), Estudios sobre la política exterior española, y $g$ ), prácticas en las Secciones del Ministerio. 


\section{Centros de Estudios Europeos}

Por la especial atención que prestan a los temas europeos, y por su ferviente adhesión al espíritu de Europa, interesa destacar el papel que podrían realizar los Centros de Estudios Europeos en el perfeccionamiento de los funcionarios. Existen en distintos países numerosos Centros de este tipo, fundados de ordinario por la iniciativa privada y consagrados a cooperar en los trabajos de aproximación de los pueblos de Europa y a crear un clima general de paz y de concordia. Dichos Institutos organizan cursos de conferencias, publicaciones, intercambios, reciben frecuentes visitas de representantes del europeísmo y están enlazados en una “Asociación de Institutos de Estudios Europeos", organismo vinculado al Centro Europeo de Cultura. En España existe un Instituto de Estudios Europeos domiciliado en Barcelona. Entre los Centros de este tipo merece citarse, por su mayor importancia, el Centro Europeo Universitario de Nancy, el Colegio de Europa de Brujas, la "Europaishes Forum" de Viena, la "Europa Archiv" de Francfort, la "Albach" del Tirol, etc.

No sería difícil organizar en estas instituciones de cultura europeístas, cursos de perfeccionamiento de los funcionarios europeos, con la garantía de que se les inculcaría un firme sentimiento de lealtad hacia Europa.

\section{INSTITUTOS DE DERECHO COMPARADO}

Otras instituciones llamadas a colaborar en el perfeccionamiento de los funcionarios europeos son los Institutos de Derecho comparado que existen en la mayoría de los países. Todos ellos están enlazados en el Comite Internacional de Derecho Comparado de la U. N. E. S. C. O.

Dada la índole de sus investigaciones y enseñanzas son especialmente aptos estos Centros para promover una aproximación entre los distintos sistemas jurfdicos que rigen las diferentes Administraciones nacionales, y suministrar conocimientos indispensa- 
bles a funcionarios que, como los europeos, tienen carácter internacional.

\section{Coloquios organizados por la Unión Europea Occidental}

Todos los años se organizan coloquios entre los altos funcionarios de los países adheridos a la Unión Europea Occidental para estudiar en común algunos temas de interés europeo. En el año en curso el coloquio se celebró en Francia, y tuvo por objeto el estudio de "El papel de funcionario administrativo y técnico en la concepción y ejecución de un plan".

\section{Proyecto de las Naciones Unidas}

En 1948, en las Naciones Unidas proyectaron crear, en colaboración con el Instituto Internacional de Ciencias Administrativas, un Centro Internacional de Altos Estudios Administrativos para la formación de los altos funcionarios nacionales e internacionales. Hasta la fecha este proyecto no se ha convertido todavía en realidad. Si llegara a fundarse, sería, naturalmente, una pieza importante para la formación y perfeccionamiento de los funcionarios europeos.

\section{Posibilidades DE ACCIÓN EN UN FUTURo INMEdiato}

Vistas las principales realizaciones producidas hasta la fecha en materia de perfeccionamiento de los funcionarios, podemos plantearnos, con vistas al futuro, una serie de cuestiones que habrán de ser objeto de deliberación en nuestro coloquio. La acertada resolución de estas cuestiones permitiría sentar las bases para la organización del perfeccionamiento de los funcionarios europeos.

Las cuestiones a estudiar son, a mi juicio, las siguientes :

1. Plan de estudios y de trabajo en los cursos de perfeccionamiento. 
2. Centros encargados de organizar los cursos de perfeccio namiento.

3. Régimen de asistencia a dichos cursos.

Vamos a considerar sumariamente cada una de estas cuestiones :

Plan de estudios y de trabajo en los cursos de perfeccionaMIENTO

La primera cuestión a considerar es la materia sobre que han de versar los cursos de perfeccionamiento de los funcionarios europeos.

Ya hemos visto que existen divergencias de criterio en cuanto a la preponderancia de la teoría y la práctica, de la cultura general y la especialización. También se discute si deben limitarse a temas de Administración pública o. han de estudiarse conjuntamente cuestiones relativas a la Administración pública y a la privada.

Será interesante conocer las. opiniones de los participantes en este coloquio acerca de este punto. Por mi parte, quiero anticipar que, a mi juicio, habría de adoptarse un sistema de equilibrio que permitiera combinar las explicaciọnes teóricas de unas pocas asignaturas, que se considerarán fundamentales, a cargo de profesores universitarios o altos funcionarios de reconocida capacidad intelectual, con las sesiones de carácter práctico destinadas a contrastar experiencias y a mejorar las técnicas de trabajo inherentes a la acción administrativa. Habría que conseguir también en estos cursos la especialización europeísta de los funcionarios, ampliando todas aquellas materias que conduzcan a un mayor conocimiento de la realidad y la unidad europeas.

En cuanto al método pedagógico, me parece importante que, junto a la disertación del profesor, exista diálogo entre éste y los asistentes al curso para aclarar las dificultades y enriquecer la explicación con las aportaciones y experiencias personales de los cursillistas.

Habrá de prestarse también atención preferente al conccimiento ¿ie la realidad social, política y económica, mediante la práctica de 
encuestas, entrevistas, visitas a Centros e instalaciones, petición de informes a expertos, etc.

En todo caso, el trabajo de los asistentes a los cursos habrá de concretarse en memorias o informes en que rindan cuenta de su labor.

En cuanto a la duración y horario de los cursos, entiende preferible el sistema inglés de "full time" y duración relativamente breve: dos o tres meses. La experiencia demuestra que son más eficaces y formativos los cursos de corta duración y máxima intensidad, que los diluídos a lo largo de un año entero con poca frecuencia de sesiones. De todos modos, en casos especiales de impasibilidad por parte de los funcionarios de dejar las funciones de st: cargo, se podrían organizar también cursos compatibles con el horario de asistencia a la oficina, de dos horas por la tarde, durante seis $u$ ocho meses.

\section{Centros encargados de organizár los cursos}

DE PERFECCIONAMIENTO

La solución ideal para el futuro podía ser la de crear un Centro superior de carácter europeo, directamente dependiente del Consejo de Europa o de otro alto organismo europeísta. Evidentemente, cuando las organizaciones europeas adquieran el arraigo que es,de esperar y el grado de desarrollo que el tiempo habrá de permitirle, será considerable el número de sus funcionarios y podrá merecer la pena tener un Centro propio para el perfeccionamiento de esos funcionarios.

Pero en el momento presente las Administraciones europeas podrían atender al perfeccionamiento de sus funcionarios sin necesidad de crear ningún Centro permanente con todo el aparato de organización, profesorado y personal consiguiente, recurriendo a la cooperación de las Universidades y otros establecimientos de enseñanza superior, de modo particular los especializados en la formación de los funcionarios.

Esta cooperación con los Centros de enseñanza ya existentes 
tendría además la ventaja de permitir la celebración de cursos de perfeccionamiento en distintos lugares de Europa. Los funcionarios europeos podrían acudir al Centro más próximo o a aquel que les of reciera mayor interés por brindarles el conocimiento de un país para ellos poco conocido, con lo que adquiriría un espíritu más abierto y se facilitaría la comprensión entre distintos pueblos de Europa.

Entre los Centros de enseñanza que podrían organizar cursos de perfeccionamiento en colaboración con las distintas Administraciones europeas están, naturalmente, en primer lugar, las Universidades. En el magnífico "rapport» de Mr. Chatenet se propugna muy acertadamente que debe conseguirse de las Universidades que incluyan, en la formación que ellas dan, elementos adecuados para preparar para el ejercicio de funciones europeas. Estos elementos pueden consistir, bien en la creación de nuevas enseñanzas especializadas, bien en orientación que se dé a otras existentes, aparte de dichas enseñanzas.

De la propuesta de Mr. Chatenet, yo me permito subrayar el interés del primer punto: es decir, la creación de nuevas enseñanzas especializadas con vistas a la formación y perfeccionamiento de los funcionarios europeos. Sería del máximo interés que las Universidades adquirieran conciencia de la magnitud del fenómeno europeísta y se dispusieran a preparar a sus alumnos para servir a las funciones europeas.

Ahora bien, esta preparación de alumnos universitarios para el servicio de las Administraciones europeas no habría de limitarse a su primera causa : es decir, a la formación previa al ingreso en una Administración europea, sino que debería abarcar también cursos de perfeccionamiento para quienes posean ya la condición de funcionarios europeos. Estos cursos habrían de darse de acuerdo con las Administraciones europeas interesadas, y según los planes y directrices guse se aprueben en el presente coloquio.

Hay que tener en cuenta, como recuerda también el profesor Chatenet, que en Europa existen dos tipos de Uriversidades : públicas y privadas. Será, pues, interesante quie la organización de las cuirsos de perfeccionamiento no se limite a las Universidades 
oficiales, sino que también se extienda a las Universidades privadas. Es más : en estos casos resultará más fácil la organización de estas enseñanzas y trabajos de carácter práctico en Universidades privadas, que tienen mayor libertad de iniciativa y facilidad de acomodación a las necesidades del momento presente.

La Universidad oficial tíene una organización 'ordinariamente rígida, con un plan de estudios unitario aprobado por el Ministero de Educación Nácional, y sin su aprobación no pueden introducirse nuevas cátedras ni enseñanzas en ninguna Universidad. Las Facultades tienen un plan de estudios común y el número de éstas en cada Universidad está regulado también por la Ley. Así ocurre, por ejemplo, en Francia y en España. Las Universidades privadas, en cambio, se apartan del molde legalista y pueden, con amplia autonomía, crear Facultades de Administración u organizar cursos especiales de perfeccionamiento de los funcionarios europeos.

Lo dicho para las Universidades vale en cierto modo para los Centros de formación de funcionarios existentes en distintos paises de Europa, para los Institutos de Derecho comparado u otros establecimientos análogos de los que se hacen referencia anteriormente.

Para la validez de los diplomas concedidos en los cursos de perfeccionamiento de los funcionarios europeos o para el.reconocimiento de dichos cursos como mérito para el ascenso de los funcionarios, podía establecerse la condición de que el programa y organización del curso se sometiera por parte de la Universidad o Centro interesado a la aprobación de la Administración europea correspondiente, aprobación que estaría sujeta al cumplimiento de determinados requisitos en orden al número y titulación de los profesores y materias fundamentales incluldas en el plan de estudios.

\section{LOPEZ RODO}

Catedráliço de Derecho administrativo 